\title{
Filter Paper Inspired Zinc Oxide Nanomaterials with High Photocatalytic Activity for Degradation of Methylene Orange
}

\author{
Huang Liu, Yanhua Zhang, Hongtao Yang, Wei Xiao, and Lanlan Sun \\ Research Institute for New Materials Technology, Chongqing Key Laboratory of Environmental Materials \& Remediation Technologies, \\ Chongqing University of Arts and Science, Chongqing 402160, China
}

Correspondence should be addressed to Yanhua Zhang; zyhcoco@163.com and Wei Xiao; showame@aliyun.com

Received 23 January 2016; Revised 5 May 2016; Accepted 17 May 2016

Academic Editor: Ewa Schab-Balcerzak

Copyright (C) 2016 Huang Liu et al. This is an open access article distributed under the Creative Commons Attribution License, which permits unrestricted use, distribution, and reproduction in any medium, provided the original work is properly cited.

\begin{abstract}
Nanometer-sized zinc oxide $(\mathrm{ZnO})$ has been synthesized through sol-gel method with natural cellulose substance (commercial filter paper) as template. The structure of zinc oxide nanomaterial was characterized by nitrogen adsorption-desorption and XRD. The morphology was observed by SEM and TEM. The results show that the hexagonal wurtzite phase is actually the only crystal phase in the sample and the product faithfully inherits the hierarchical morphology and the complex network structure of the original filter paper, which is composed of many randomly intersecting zinc oxide microfibers and nanosheets with lots of close stacked particles adsorbed on the surface. Moreover, these zinc oxide nanomaterials possess abundant mesoporous structure with an average pore diameter of $c a .21 \mathrm{~nm}$ and a wide pore size distribution $(3-30 \mathrm{~nm})$. Due to the strong absorption ability in the UV range, the zinc oxide nanomaterial prepared by this method displays significantly high photocatalytic activity for degrading methyl orange. In a short period of 20 minutes, the zinc oxide nanomaterial has degraded about $50 \%$ of the original MO, and the MO dye is fully degraded after UV irradiation for 80 minutes.
\end{abstract}

\section{Introduction}

In recent years, the environmental pollution has been increasing. The photocatalytic processes in polluted air and water through photocatalytic oxidation of semiconductor catalysts have been studied extensively in recent years [1-3]. Anatase titania has been widely used as a catalyst both in liquid and in gaseous systems showing good performances concerning photoreactivity and photostability [4-6]. Zinc oxide is a direct wide band gap $(3.37 \mathrm{eV})$ oxide semiconductor photocatalyst, which is close to band gap energy of $\mathrm{TiO}_{2}(3.2 \mathrm{eV})$, and the mechanism of photocatalysis has been proven to be similar to that of $\mathrm{TiO}_{2}$ [7]. Nanometer-sized zinc oxide as an important inorganic function nanomaterial was widely used in rubber [8], coat [9], catalyst [10], and electronic engineering fields [11] due to its unique properties such as high photosensitivity, transparency in the visible, high infrared reflectivity, acoustic characteristics, high electrochemical stability, and excellently electronic properties. The dispersion and surface area of zinc oxide, which depend on the synthesis method, are important factors for determining its photocatalytic activity [12-14]. Zinc oxide nanomaterials can be prepared by various methods, such as alkali precipitation [15], pulsed laser deposition [16], magnetron sputtering [17], chemical vapor deposition [18], and sol-gel processing [19-21]. However, among all of these techniques, the sol-gel process is one of the versatile methods to prepare various morphologies nanosized zinc oxide without complicated instruments. The most important advantages are the simplicity of equipment, the ability to accurately control stoichiometry, high homogeneity, and relatively low process temperature. Therefore, sol-gel technique could be a suitable method for the preparation of various morphologies nanomaterials.

Synthesis by using natural materials as template is a facile strategy to yield functional materials with complex and hierarchical natural structures, which are generally difficult to prepare even through the most advanced synthetic methods. Among these biotemplates, natural cellulosic substances such as the common filter paper possess a macroscopic-tonanoscopic random morphological hierarchy. Using these common and low-cost natural cellulose substances as 
the structural templates, many nanomaterials with complex functional nanostructures can be formed. With the natural cellulosic materials as structural templates, unique and intricate morphologies originated from nature substances become the choice for the design and preparation of zinc oxide nanomaterials, which extensively expands the structural varieties of $\mathrm{ZnO}$ materials.

In this paper, we synthesized nanometer-sized zinc oxide through sol-gel method with natural cellulose substance (commercial filter paper) and polyethylene glycol (PEG) as dual templates, $\mathrm{Zn}\left(\mathrm{CH}_{3} \mathrm{COO}\right)_{2} \cdot 2 \mathrm{H}_{2} \mathrm{O}$ as precursor, ethanol as solvent, and $\mathrm{NH}\left(\mathrm{C}_{2} \mathrm{H}_{2} \mathrm{OH}\right)_{2}$ as chelating agent. The resulting zinc oxide nanomaterials faithfully inherit the hierarchical morphologies and the complex network structures of the initial cellulose substance and exhibit relatively high photocatalytic activity.

\section{Experimental}

2.1. Reagents. Pure cellulosic fiber from commercial ashless quantitative filter paper (GB/T1914-93) was used for the entire process. Polyethylene glycol $\left[M=2000, \mathrm{H}\left(\mathrm{OCH}_{2} \mathrm{CH}_{2}\right)_{n} \mathrm{OH}\right.$, PEG, AR], zinc acetate $\left[M=219.50, \mathrm{Zn}\left(\mathrm{CH}_{3} \mathrm{COO}\right)_{2} \cdot 2 \mathrm{H}_{2} \mathrm{O}\right.$, $\mathrm{AR}$ ], and diethanolamine $\left(M=105.14, \mathrm{NH}\left(\mathrm{C}_{2} \mathrm{H}_{2} \mathrm{OH}\right)_{2}\right.$, DEA, AR) were purchased from Aldrich. All the other chemicals were guaranteed ones and used as received. Milli-Q water (resistivity, 18.2 $\mathrm{M} \Omega \mathrm{cm}^{-1}$ ) was used in all related cases.

2.2. Preparation of Zinc Oxide Nanomaterials. Zinc oxide sol was prepared in the following way: Firstly, zinc acetate $\left[\mathrm{Zn}\left(\mathrm{CH}_{3} \mathrm{COO}\right)_{2} \cdot 2 \mathrm{H}_{2} \mathrm{O}\right]$ was dissolved in $50 \mathrm{~mL}$ of absolute ethanol and stirred with a magnetic stirrer $\left(70^{\circ} \mathrm{C}\right)$ to be mixed thoroughly. When the solution changed into an emulsion, an amount of diethanolamine $\left[\mathrm{NH}\left(\mathrm{C}_{2} \mathrm{H}_{2} \mathrm{OH}\right)_{2} ; \mathrm{DEA}\right]$ as chelating agent was added to the emulsion (the molar ratio of DEA/zinc acetate was $1: 1)$. When the emulsion became clear after a period of stirring, $0.7 \mathrm{~g}$ polyethylene glycol (PEG) was added and continually stirred for $2 \mathrm{~h}$ until a transparent sol was obtained. After the sol solution was cooled to the room temperature, $150 \mathrm{mg}$ precutted squared filter paper fractions of $c a .5 \mathrm{~mm} \times 5 \mathrm{~mm}$ were immersed in the sol solution for $3 \mathrm{~h}$ and then the filter paper fractions were filtered off, washed with absolute ethanol, and finally vacuum-dried at room temperature overnight. The as-prepared filter paper was firstly calcined in a tubular furnace at $100^{\circ} \mathrm{C}$ for $1 \mathrm{~h}$ and then heated up to $500^{\circ} \mathrm{C}$ and kept for $3 \mathrm{~h}$. The whole process was in air atmosphere with a ramping rate of $2^{\circ} \mathrm{C} / \mathrm{min}$.

2.3. Characterizations. The morphologies of samples were observed by a field emission scanning electron microscope (FE-SEM, Hitachi, S-4800), transmission electron microscopy (TEM), and high-resolution transmission electron microscopy (HR-TEM, FEI Tecnai G20/JEM 2010, operated at $200 \mathrm{kV}$ ). To prepare the specimens for SEM and TEM observation, a small piece of the sample sheet specimen was suspended in ethanol by ultrasonication for several seconds. The suspension was dropped onto silicon wafer followed by sputtering with gold or platinum to increase conductivity for
SEM observation or onto copper mesh for TEM observation, and the specimens were dried in air. Crystal phase of the obtained materials was determined by powder X-ray diffraction (XRD) recorded on a Dandong TD3500 Advanced Diffractometer with $\mathrm{Cu}-\mathrm{K} \alpha$ radiation $(\lambda=0.15418 \mathrm{~nm})$ and scan rate $0.05^{\circ} 2 \theta / \mathrm{s}$ and within range of $2 \theta$ from $20^{\circ}$ to $90^{\circ}$ at room temperature. The nitrogen adsorption and desorption isotherms were measured at $77 \mathrm{~K}$ on a TriStar II 3020 surface area and pore analyzer. The linear part of the Brunauer-Emmett-Teller (BET) equation was used for the specific surface area determination. The pore size distributions were obtained from the adsorption branch of the nitrogen physisorption isotherms using the BJH method. Ultraviolet-visible diffuse reflectance (UV-vis DRS) absorption spectra were tested using a U-4100 spectrometer (Hitachi, Japan) with $\mathrm{BaSO}_{4}$ powder as the reference standard.

2.4. Photodegradation Experiment. Methyl orange $\left(\mathrm{C}_{14} \mathrm{H}_{14} \mathrm{~N}_{3} \mathrm{NaO}_{3} \mathrm{~S}\right)$, a widely used dye, was employed as a representative dye pollutant to evaluate the photocatalytic activity of zinc oxide nanomaterials under UV light. Firstly, $100 \mathrm{~mL}$ aqueous solution of zinc oxide catalyst $(0.2 \mathrm{~g})$ was treated with ultrasonication for $30 \mathrm{~min}$ to promote dispersion uniformity and $400 \mathrm{~mL}$ dye aqueous solution of $\mathrm{MO}$ $(30 \mathrm{mg} / \mathrm{L})$ was prepared for subsequent photocatalytic activity test. Then, the two prepared solutions above were mixed in a quartz tube. Before irradiation, the above mixed solution was stirred for $30 \mathrm{~min}$ in the dark to ensure the establishment of adsorption-desorption equilibrium. Under ambient conditions and stirring, the quartz tube was exposed to the $\mathrm{UV}$ irradiation produced by a $500 \mathrm{~W} \mathrm{Hg}$ arc lamp equipped with a band-pass light filter $(365 \pm 15 \mathrm{~nm})$. At selected time intervals, about $3 \mathrm{~mL}$ of the suspension was withdrawn for analysis on a Varian UV-vis spectrophotometer (Cary-50, Varian Co.). The percentage of residual dye is reported as $C / C_{0}$. Here, $C$ is the concentration of dye solution at each irradiated time interval, and $C_{0}$ is the initial concentration of dye solution before photodegradation experiment.

\section{Results and Discussion}

3.1. Characterization of Zinc Oxide Nanomaterials. The XRD pattern of the obtained zinc oxide nanomaterials demonstrates that hexagonal wurtzite phase is actually the only crystal phase in the product (Figure 1). The distinctive X-ray diffraction peaks around $31.8^{\circ}, 34.4^{\circ}, 36.3^{\circ}, 47.5^{\circ}, 56.6^{\circ}, 62.8^{\circ}$, $66.4^{\circ}, 67.9^{\circ}$, and $69.1^{\circ}$ correspond to the hexagonal wurtzite phase (100), (002), (101), (102), (110), (103), (200), (112), and (201) crystalline planes (I41/amd, JCPDF card number 653411).

The widening XRD peak enables estimation of the crystallite size via the Scherrer equation as below:

$$
d=\frac{k \lambda}{\beta \cos (\theta)},
$$

where $\beta$ (radian) is the full-width at half-maximum for the peak at $2 \theta=36.3^{\circ} ; k$ is a constant $(0.89) ; \lambda$ is the $\mathrm{X}$-ray wavelength (1.5418 $\AA$ for $\mathrm{Cu} \mathrm{K} \alpha)$; $d$ is the particle diameter; and $\theta$ is 


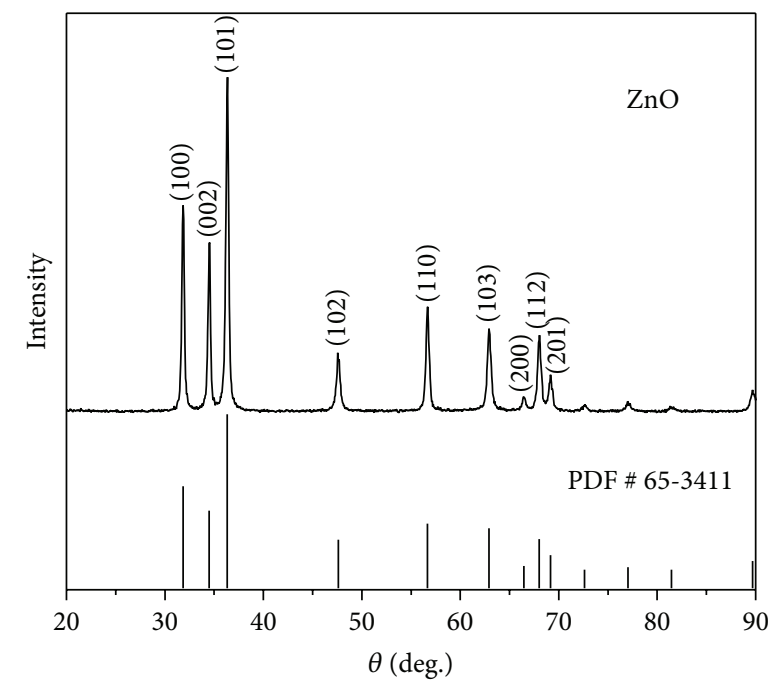

Figure 1: X-ray diffraction pattern of the prepared filter paper inspired zinc oxide nanomaterial.

the angle of the diffraction peak (degree). This analysis yields an estimate of $c a .25 .3 \mathrm{~nm}$ for the average crystallite size based on the (101) peak.

The morphology of zinc oxide product was investigated by SEM and TEM, and typical images are shown in Figure 2. Figure 2(a) is the overview FE-SEM image of the sample, which shows that the zinc oxide sample faithfully inherits the hierarchical morphology and the complex network structure of the original filter paper template, and the resulting zinc oxide is composed of many randomly intersecting zinc oxide microfibers and nanosheets with lots of particles adsorbed on the surface. With further magnification, we can observe that the solid zinc oxide microtubes and nanosheets are composed of lots of closely packing zinc oxide particles (Figure 2(b)). Figure 2(c) is a typical TEM image of zinc oxide nanosheet, in which the solid nanosheet structure of sample is clearly identified and the zinc oxide particles with diameters of $c a$. $28 \mathrm{~nm}$ are measured. The HR-TEM image (Figure 2(d)) shows that the measured lattice spacing of sample is $0.281 \mathrm{~nm}$, which corresponds to the lattice distance of hexagonal wurtzite (100) phase and confirms the formation of hexagonal wurtzite nanoparticles. Several multicrystal diffraction rings to be indexed to the anatase phase can be observed obviously in the SAED pattern of sample (Figure $2(d)$ insert), in particular, (from small to large rings) indexed as $100(d=0.281 \mathrm{~nm}), 002$ $(d=0.26 \mathrm{~nm}), 101(d=0.247 \mathrm{~nm}), 102(d=0.191 \mathrm{~nm}), 110$ $(d=0.162 \mathrm{~nm}), 103(d=0.147 \mathrm{~nm})$, and $200(d=0.141 \mathrm{~nm})$. This result is in good agreement with XRD analysis.

The nitrogen adsorption and desorption curve of the synthesized zinc oxide nanomaterials is shown in Figure 3. The hysteresis loops of $\mathrm{H} 4$ type proved the existence of mesopores, which was observed in the case of aggregates of particles giving rise to pores. The hysteresis loop at a relative pressure $\left(P / P_{0}\right)$ between 0.5 and 0.95 is attributed to the stacking of zinc oxide particles. The capillary condensation step at relative pressure about $0.8\left(P / P_{0}\right)$ is a contribution of the typical mesopores in the sample. The sharp capillary condensation step occurs in the relative pressure range of $0.8-1.0\left(P / P_{0}\right)$ due to the existence of mesopores in the materials. The pore distribution plot of the synthesized materials was determined by the BJH (Barrett-Joyner-Halenda) method from the desorption branch of isotherm as shown in the inset of Figure 3, which indicates that the synthesized zinc oxide has macropores with an average pore diameter of $\sim 21 \mathrm{~nm}$ and a wide pore size distribution. The BET specific surface area of the synthesized zinc oxide is estimated to be about $14.4293 \mathrm{~m}^{2} / \mathrm{g}$ and the total pore volume is about $0.119175 \mathrm{~cm}^{3} / \mathrm{g}$.

The UV-vis adsorption spectrum of the prepared zinc oxide nanomaterials was measured by UV-vis optical absorbance pattern (Figure 4(a)), showing that the broad absorption region mainly concentrated in less than $400 \mathrm{~nm}$, which indicates that this sample is mainly to absorb ultraviolet light. Semiconductors are classified as either direct or indirect according to the lowest allowed electronic transition [22]. As we all know the zinc oxide crystal is a direct semiconductor and its absorption index $\alpha(h \nu)$ should follow the $\left(h v-E_{g}\right)^{3 / 2}$ dependence. Normally nanomaterials exhibit an absorption spectrum such as that of the direct band gap semiconductor, and its absorption coefficient $\alpha(h \nu)$ follows the $\left(h \nu-E_{g}\right)^{1 / 2}$ law. So, the relationship between the absorption coefficient $(\alpha)$ and incident $p$ photon energy $(h \nu)$ can be written as [23]

$$
\begin{aligned}
\alpha & =\frac{B_{d}\left(h v-E_{g}\right)^{1 / 2}}{h v} \Longrightarrow \\
\left(\frac{\alpha h v}{B_{d}}\right)^{2} & =h v-E_{g} \Longrightarrow \\
(A h v)^{2} & =h v-E_{g},
\end{aligned}
$$

where $B_{d}$ is the absorption constant for direct transitions [24]. Therefore, the band gap energy can be estimated from a plot of $(\alpha h v)^{2}$ versus the photon energy $(h v)$. As the absorbance $(A)$ is proportional to the absorption coefficient $(\alpha), A$ can substitute for $\alpha$. Plots of $(A h \nu)^{2}$ versus the photon energy $(h \nu)$ are shown in Figure 4(b). The intercept of the tangent to the plot gives a good approximation of the direct band gap energies of the samples. Extrapolating the linear part of the curve for the zinc oxide sample gives a direct band gap of $3.18 \mathrm{eV}$, which is narrower than commercial zinc oxide semiconductor $(3.37 \mathrm{eV})$. The reduction of band gap value for the synthesized $\mathrm{ZnO}$ nanomaterials here should arise from the structural and electronic defects such as interstitials and vacancies created during synthesis process. In the sample, the band gap was obtained from the electronic transition between the filled valence states to the energy level of the generated defects instead of the transition between the valence band and the conduction band as usual and resulted in a lower band gap value than the bulk $\mathrm{ZnO}$ with the perfect structure [25].

The results above indicate that our zinc oxide samples possess stronger capability of absorption than commercial zinc oxide semiconductor in the ultraviolet light range.

3.2. Photocatalytic Degradation of MO. The photocatalytic activity of our porous zinc oxide sample was further evaluated 


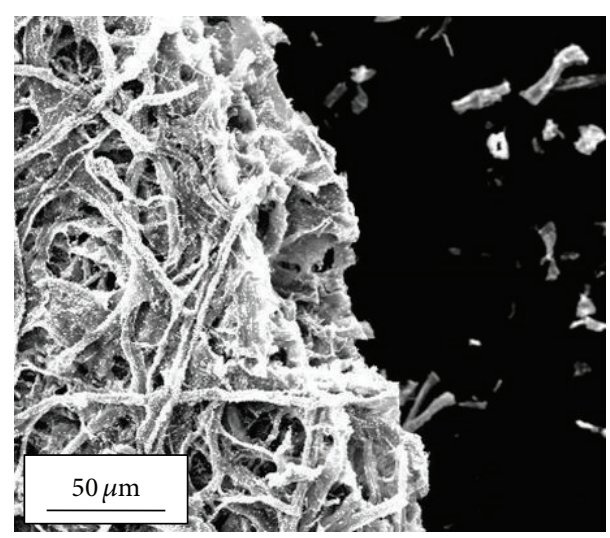

(a)

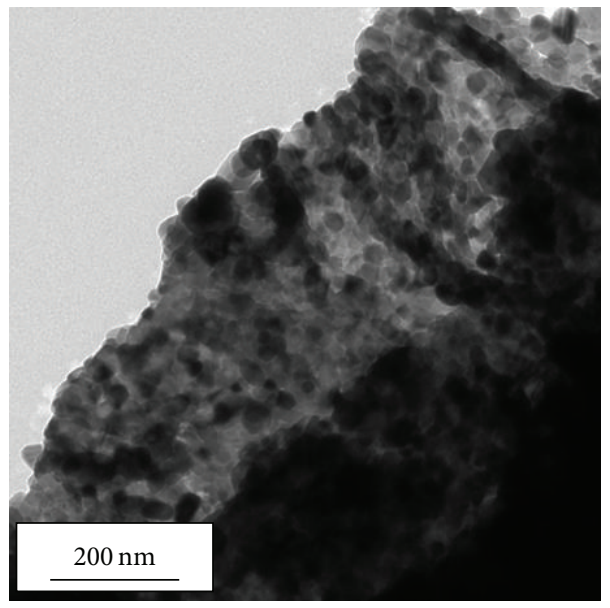

(c)

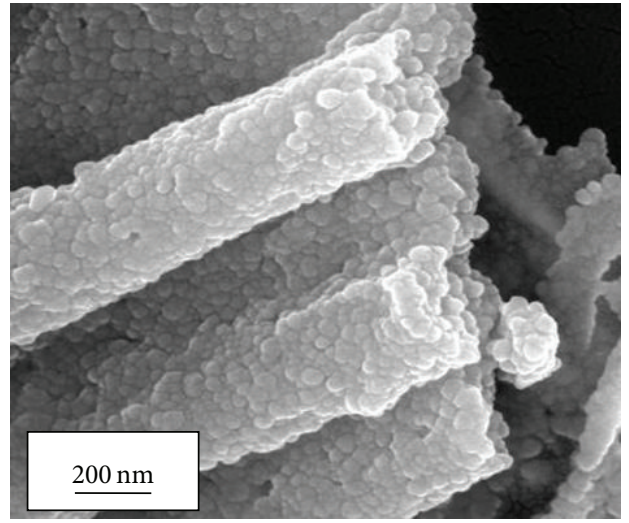

(b)

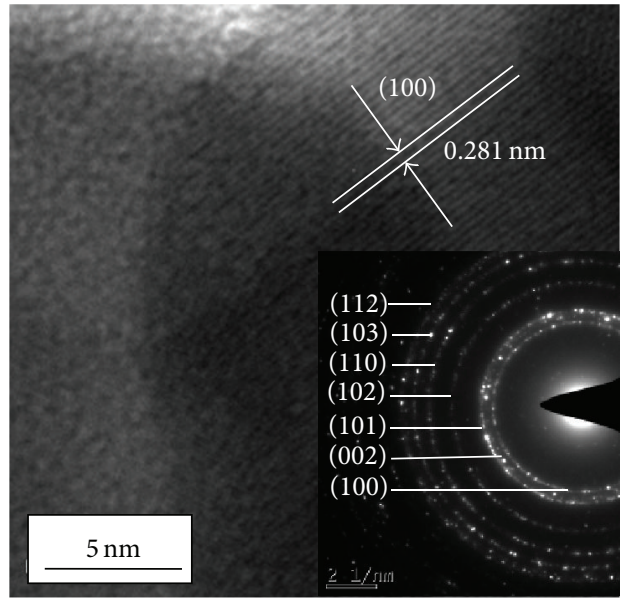

(d)

FIGURE 2: Micromorphology observation of prepared zinc oxide nanomaterials. (a) FE-SEM image, (b) further magnified SEM image, (c) typical TEM image of zinc oxide nanosheet, and (d) HR-TEM image and SAED pattern (insert).

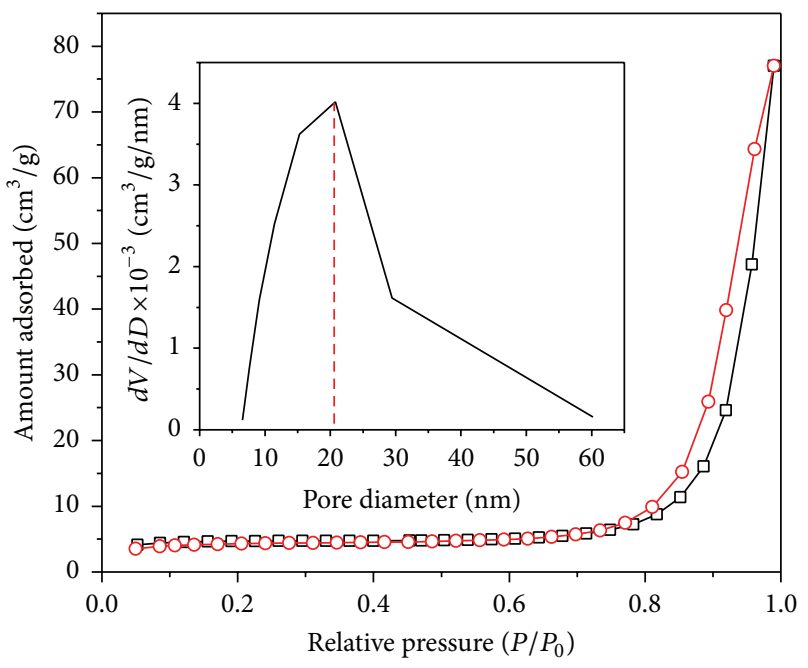

FIGURE 3: $\mathrm{N}_{2}$ adsorption/desorption isotherms of the prepared zinc oxide nanomaterials and $\mathrm{BJH}$ pore size distribution plot (insert).

by monitoring the degradation of methyl orange (MO) under UV irradiation (Figure 5). Before UV irradiation, the mixture containing the catalyst and MO was stirred in the dark for $30 \mathrm{~min}$ to ensure that $\mathrm{MO}$ was adsorbed to saturation on the surface of catalysts. With the time increasing, gradually weakening orange liquid can be observed in Figure 5(a), implying the decrease of MO concentration in mixture. Figure 5(b) is the evolution of the corresponding $C / C_{0}$ with $\mathrm{UV}$ exposure time of our $\mathrm{ZnO}$ nanomaterials. It can be observed that the zinc oxide sample exhibits relatively high photocatalytic activity. With the currently developed zinc oxide nanomaterial as photocatalyst, it only took $20 \mathrm{~min}$ and $40 \mathrm{~min}$ to degrade $50 \%$ and $80 \%$ of original MO under UV irradiation, respectively, and the MO dye was totally degraded within $80 \mathrm{~min}$. However, under almost the same photocatalytic condition as that in our experiments, the $\mathrm{ZnO}$ nanowires synthesized by $\mathrm{Niu}$ et al. only degraded less than $20 \%$ of the original MO within 20 minutes and the degradation ratio of MO was no more than $40 \%$ after UV irradiation for 80 minutes [26]. In addition, for the $\mathrm{ZnO}$ film prepared by Yin et al., the degradation ratios of $\mathrm{MO}$ were only $40 \%$ and $58 \%$ after photocatalytic reaction under UV irradiation for $20 \mathrm{~min}$ and $40 \mathrm{~min}$, respectively [27]. Apparently, the photocatalytic efficiency of such reported 


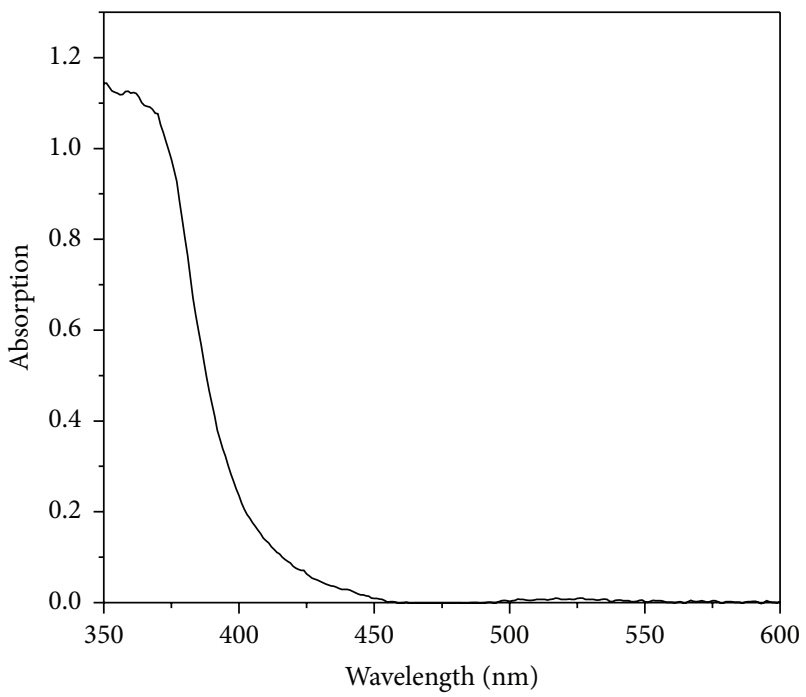

(a)

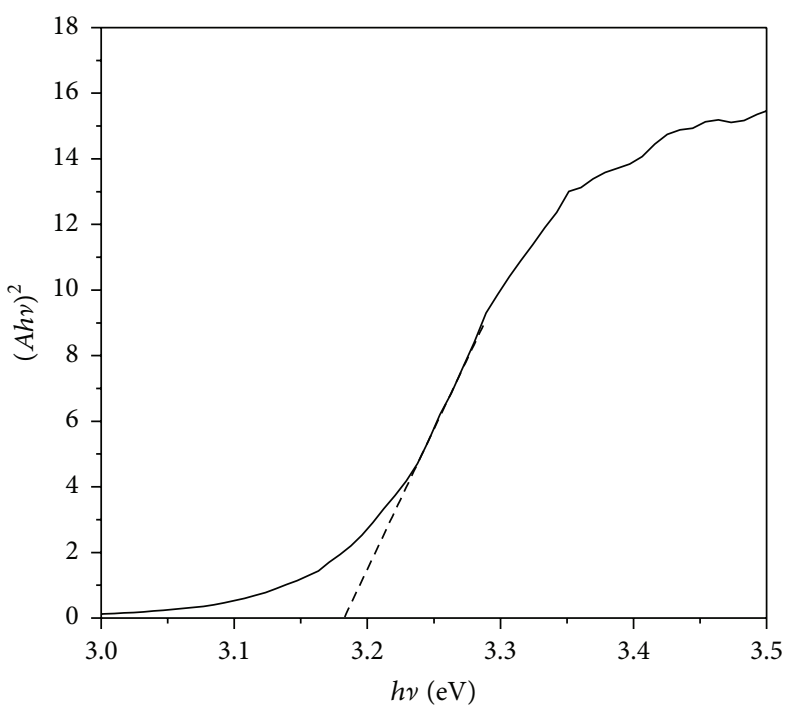

(b)

FIGURE 4: (a) UV-vis absorption spectra of the prepared zinc oxide nanomaterials and (b) the plots of $(A h \nu)^{2}$ versus the photon energy $(h \nu)$.

ZnO-based photocatalysts is much lower than that of the currently developed filter paper inspired $\mathrm{ZnO}$ nanomaterials, suggesting their superior photocatalytic activity.

The kinetics of photocatalytic degradation of MO can be depicted by pseudo-first-ordered kinetics equation as below [28]:

$$
\ln \left(\frac{C}{C_{0}}\right)=-k t
$$

where $k$ is the pseudo-first-order rate constant $\left(\min ^{-1}\right), C_{0}$ is initial concentration, and $C$ is the concentration of $\mathrm{MO}$ at time $t$ (min). Figure 5(c) shows the plot of $\ln \left(C / C_{0}\right)$ versus time, in which linear plots were observed with $R^{2}$ values equal to 0.96168 . The result indicates that photodegradation of $\mathrm{MO}$ obeys pseudo-first-order kinetics and the calculated pseudofirst-order rate is $0.04734 \mathrm{~min}^{-1}$.

Figure 5 shows that the filter paper inspired zinc oxide nanomaterials exhibit relatively high photocatalytic activity. Though these zinc oxide samples possess a low surface area $\left(14.4293 \mathrm{~m}^{2} / \mathrm{g}\right)$ and weak adsorptive capacity in the dark adsorption of photocatalytic test (Figure 5(b)), they still exhibit relatively efficient photocatalytic rates during the photochemical process, which contribute from its stronger absorption ability in the UV range.

\section{Conclusion}

In this paper, $\mathrm{ZnO}$ porous thin films were prepared on natural cellulose substance (commercial filter paper) by sol-gel method with polyethylene glycol (PEG) as organic template, $\mathrm{Zn}\left(\mathrm{CH}_{3} \mathrm{COO}\right)_{2} \cdot 2 \mathrm{H}_{2} \mathrm{O}$ as precursor, ethanol as solvent, and $\mathrm{NH}\left(\mathrm{C}_{2} \mathrm{H}_{2} \mathrm{OH}\right)_{2}$ as chelating agent. The obtained material was characterized by XRD, SEM, TEM, SAED, $\mathrm{N}_{2}$ adsorption/desorption, and UV-vis. From the results of the XRD and SAED, we can deduce that hexagonal wurtzite phase is actually the only crystal phase in the sample. The SEM results show that the product faithfully inherits the hierarchical morphologies of the initial cellulose substances and consists of many randomly intersecting zinc oxide microfibers and nanosheets with lots of particles adsorbed on the surface. With further magnification, we can observe that the solid zinc oxide microtubes and nanosheets are composed of lots of closely packing zinc oxide particles. Analysis of TEM and $\mathrm{N}_{2}$ adsorption/desorption isotherm plots show that these zinc oxide nanomaterials possess the mesoporous structure with an average pore diameter of $c a .21 \mathrm{~nm}$ and a wide pore size distribution (3-30 nm). The photocatalytic activity for methyl orange degradation was tested under UV irradiation. The result shows the zinc oxide nanomaterial prepared by this method displays significantly high photocatalytic activity due to its stronger absorption ability in the UV range. Under the UV irradiation in a short period of 20 minutes, the zinc oxide nanomaterials have degraded about $50 \%$ of the original $\mathrm{MO}$, and all of the dye is almost fully degraded after UV irradiation for 80 minutes. The current facile but effective method, which uses the common commercial filter paper as structural template, will reduce the production cost of nanomaterials, expand the structural varieties of $\mathrm{ZnO}$ materials, and provide a straightforward approach to fabricate other metal oxides with distinct and complicated structures and morphologies.

\section{Competing Interests}

The authors declare that there are no competing interests regarding the publication of this paper.

\section{Acknowledgments}

This work was financially supported by the National Natural Science Foundation of China (21101136, 21401015), Project of International Cooperation and Exchanges NSFC (no. 


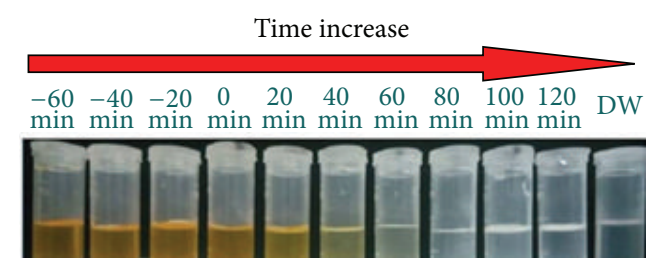

(a)

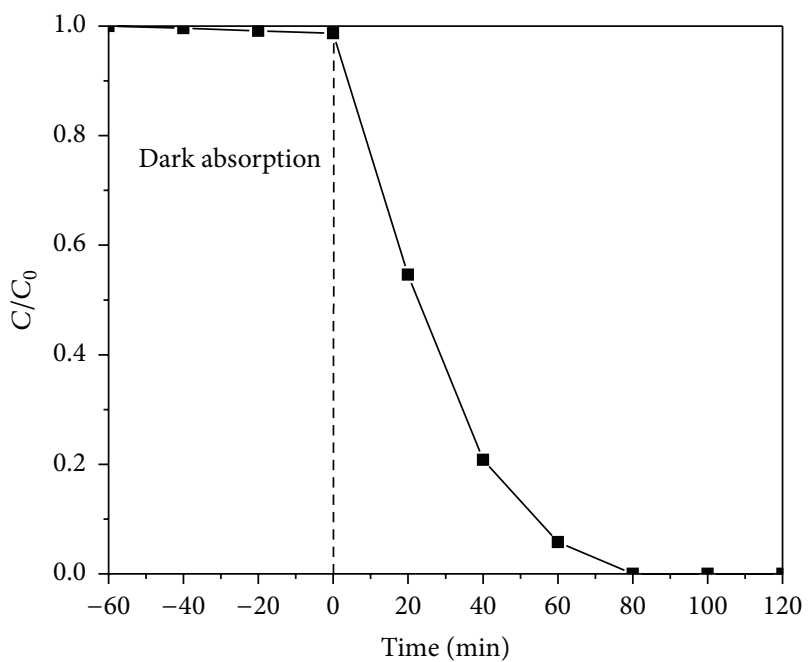

(b)

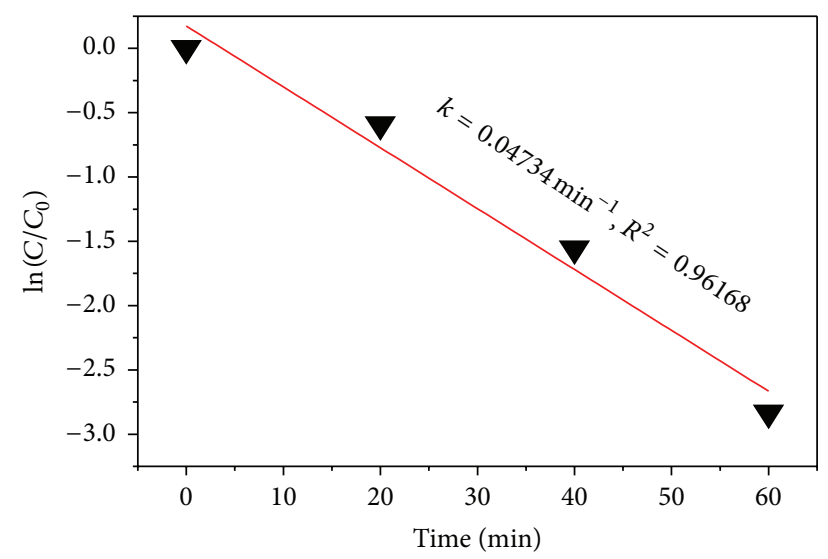

(c)

FIgURE 5: (a) Photographs of MO under UV irradiation at different times, (b) liquid-phase photocatalytic degradation of MO under the irradiation of UV light, and (c) kinetics of photocatalytic degradation of MO under UV for filter paper inspired zinc oxide nanomaterials.

21310102011), the Key Project of Chinese Ministry of Education (212144), Natural Science Foundation Project of CQ CSTC (cstc2012jjA50037, cstc2014jcyjA50012), and Chongqing University of Arts and Sciences (nos. R2012CJ15, R2013CJ04).

\section{References}

[1] H. Tong, S. Ouyang, Y. Bi, N. Umezawa, M. Oshikiri, and J. Ye, "Nano-photocatalytic materials: possibilities and challenges," Advanced Materials, vol. 24, no. 2, pp. 229-251, 2012.
[2] R. Marschall, "Semiconductor composites: strategies for enhancing charge carrier separation to improve photocatalytic activity," Advanced Functional Materials, vol. 24, no. 17, pp. 24212440, 2014

[3] I. Paramasivam, H. Jha, N. Liu, and P. Schmuki, "A review of photocatalysis using self-organized $\mathrm{TiO}_{2}$ nanotubes and other ordered oxide nanostructures," Small, vol. 8, no. 20, pp. 30733103, 2012.

[4] A.-Y. Zhang, L.-L. Long, W.-W. Li, W.-K. Wang, and H.-Q. Yu, "Hexagonal microrods of anatase tetragonal $\mathrm{TiO}_{2}$ : self-directed growth and superior photocatalytic performance," Chemical Communications, vol. 49, no. 54, pp. 6075-6077, 2013.

[5] D. Yang, H. Liu, Z. Zheng et al., "An efficient photocatalyst structure: $\mathrm{TiO}_{2}(\mathrm{~B})$ nanofibers with a shell of anatase nanocrystals," Journal of the American Chemical Society, vol. 131, no. 49, pp. 17885-17893, 2009.

[6] X. Yu, B. Kim, and Y. K. Kim, "Highly enhanced photoactivity of anatase $\mathrm{TiO}_{2}$ nanocrystals by controlled hydrogenationinduced surface defects," ACS Catalysis, vol. 3, no. 11, pp. 24792486, 2013.

[7] B. Neppolian, S. Sakthivel, B. Arabindoo, M. Palanichamy, and V. Murugesan, "Degradation of textile dye by solar light using $\mathrm{TiO}_{2}$ and $\mathrm{ZnO}$ photocatalysts," Journal of Environmental Science \& Health-Part A, vol. 34, no. 9, pp. 1829-1838, 1999.

[8] A. Susanna, L. Armelao, E. Callone et al., " $\mathrm{ZnO}$ nanoparticles anchored to silica filler. A curing accelerator for isoprene rubber composites," Chemical Engineering Journal, vol. 275, pp. 245252, 2015.

[9] Y. Sun, G. M. Fuge, N. A. Fox, D. J. Riley, and M. N. R. Ashfold, "Synthesis of aligned arrays of ultrathin $\mathrm{ZnO}$ nanotubes on a $\mathrm{Si}$ wafer coated with a thin ZnO film," Advanced Materials, vol. 17, no. 20, pp. 2477-2481, 2005.

[10] P. P. Ghosh and A. R. Das, "Nanocrystalline and reusable ZnO catalyst for the assembly of densely functionalized $4 \mathrm{H}$-chromenes in aqueous medium via one-pot three component reactions: a greener 'NOSE' approach," Journal of Organic Chemistry, vol. 78, no. 12, pp. 6170-6181, 2013.

[11] H. Guo, N. Lu, J. Dai, X. C. Zeng, X. Wu, and J. Yang, "Electronic structure engineering in chemically modified ultrathin $\mathrm{ZnO}$ nanofilms via a built-in heterointerface," RSC Advances, vol. 4, no. 36, pp. 18718-18723, 2014.

[12] C. Tian, Q. Zhang, A. Wu et al., "Cost-effective large-scale synthesis of $\mathrm{ZnO}$ photocatalyst with excellent performance for dye photodegradation," Chemical Communications, vol. 48, no. 23, pp. 2858-2860, 2012.

[13] S. Polarz, A. V. Orlov, F. Schüth, and A.-H. Lu, "Preparation of high-surface-area zinc oxide with ordered porosity, different pore sizes, and nanocrystalline walls," Chemistry - A European Journal, vol. 13, no. 2, pp. 592-597, 2007.

[14] X. Bai, L. Wang, R. Zong, Y. Lv, Y. Sun, and Y. Zhu, "Performance enhancement of $\mathrm{ZnO}$ photocatalyst via synergic effect of surface oxygen defect and graphene hybridization," Langmuir, vol. 29, no. 9, pp. 3097-3105, 2013.

[15] Y.-R. Guo, F.-D. Yu, G.-Z. Fang, and Q.-J. Pan, "Synthesis, structural characterization and photoluminescent properties of mesoporous $\mathrm{ZnO}$ by direct precipitation with lignin-phosphate quaternary ammonium salt," Journal of Alloys and Compounds, vol. 552, pp. 70-75, 2013.

[16] Z. Qiu, H. Gong, X. Yang et al., "Phosphorus concentration dependent microstructure and optical property of $\mathrm{ZnO}$ nanowires grown by high-pressure pulsed laser deposition," The 
Journal of Physical Chemistry C, vol. 119, no. 8, pp. 4371-4378, 2015.

[17] H. Liu, P. Zhou, L. Zhang, Z. Liang, H. Zhao, and Z. Wang, "Effects of oxygen partial pressure on the structural and optical properties of undoped and $\mathrm{Cu}$-doped $\mathrm{ZnO}$ thin films prepared by magnetron co-sputtering," Materials Letters, vol. 164, pp. 509-512, 2016.

[18] S. Park, J.-W. Park, S.-M. Yang, K. Kim, and N. Hwang, "Effect of electric bias on the deposition behavior of $\mathrm{ZnO}$ nanostructures in the chemical vapor deposition process," The Journal of Physical Chemistry C, vol. 119, no. 44, pp. 25047-25052, 2015.

[19] Y. Sun, J. H. Seo, C. J. Takacs, J. Seifter, and A. J. Heeger, "Inverted polymer solar cells integrated with a low-temperature-annealed sol-gel-derived $\mathrm{ZnO}$ film as an electron transport layer," Advanced Materials, vol. 23, no. 14, pp. 1679-1683, 2011.

[20] M. Kashif, U. Hashim, M. E. Ali, K. L. Foo, and S. M. Usman Ali, "Morphological, structural, and electrical characterization of sol-gel-synthesized $\mathrm{ZnO}$ nanorods," Journal of Nanomaterials, vol. 2013, Article ID 478942, 7 pages, 2013.

[21] J. Joo, S. G. Kwon, J. H. Yu, and T. Hyeon, "Synthesis of $\mathrm{ZnO}$ nanocrystals with cone, hexagonal cone, and rod shapes via non-hydrolytic ester elimination sol-gel reactions," Advanced Materials, vol. 17, no. 15, pp. 1873-1877, 2005.

[22] X. H. Wang, J.-G. Li, H. Kamiyama et al., "Pyrogenic iron(III)doped $\mathrm{TiO}_{2}$ nanopowders synthesized in RF thermal plasma: phase formation, defect structure, band gap, and magnetic properties," Journal of the American Chemical Society, vol. 127, no. 31, pp. 10982-10990, 2005.

[23] J. Serrano, A. H. Romero, F. J. Manjon et al., "Pressure dependence of the lattice dynamics of $\mathrm{ZnO}$ : an ab initio approach," Physical Review B: Condensed Matter and Materials Physics, vol. 69, Article ID 094306, 2004.

[24] E. Mooser and W. B. Pearson, Progress in Semiconductor, vol. 5, Heywood and Co., London, UK, 1960.

[25] S. Suwanboon, P. Amornpitoksuk, and N. Muensit, "Dependence of photocatalytic activity on structural and optical properties of nanocrystalline $\mathrm{ZnO}$ powders," Ceramics International, vol. 37, no. 7, pp. 2247-2253, 2011.

[26] H. Niu, X. Zhao, L. Duan et al., "Influence of annealing ambient on the structure, photoluminescence and photocatalytic activity of low temperature grown $\mathrm{ZnO}$ nanowires," Superlattices and Microstructures, vol. 83, article 3561, pp. 71-77, 2015.

[27] B. Yin, S. Zhang, D. Zhang et al., "ZnO film photocatalysts," Journal of Nanomaterials, vol. 2014, Article ID 186916, 7 pages, 2014.

[28] L.-Y. Yang, S.-Y. Dong, J.-H. Sun, J.-L. Feng, Q.-H. Wu, and S.P. Sun, "Microwave-assisted preparation, characterization and photocatalytic properties of a dumbbell-shaped $\mathrm{ZnO}$ photocatalyst," Journal of Hazardous Materials, vol. 179, no. 1-3, pp. 438443, 2010. 

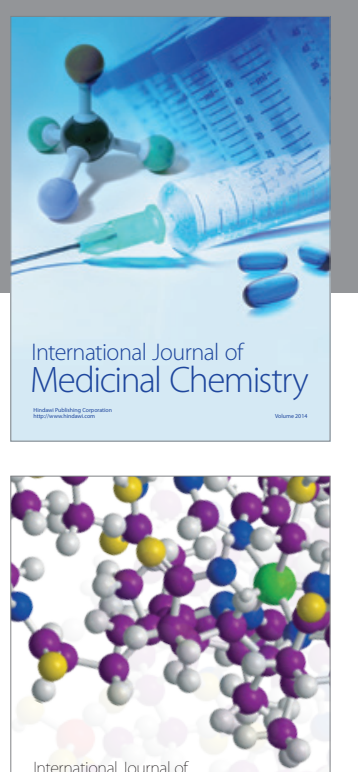

Carbohydrate Chemistry

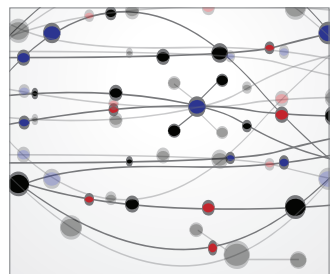

The Scientific World Journal
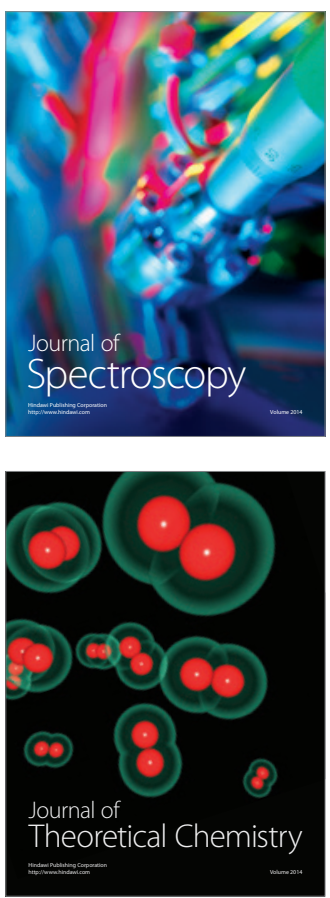
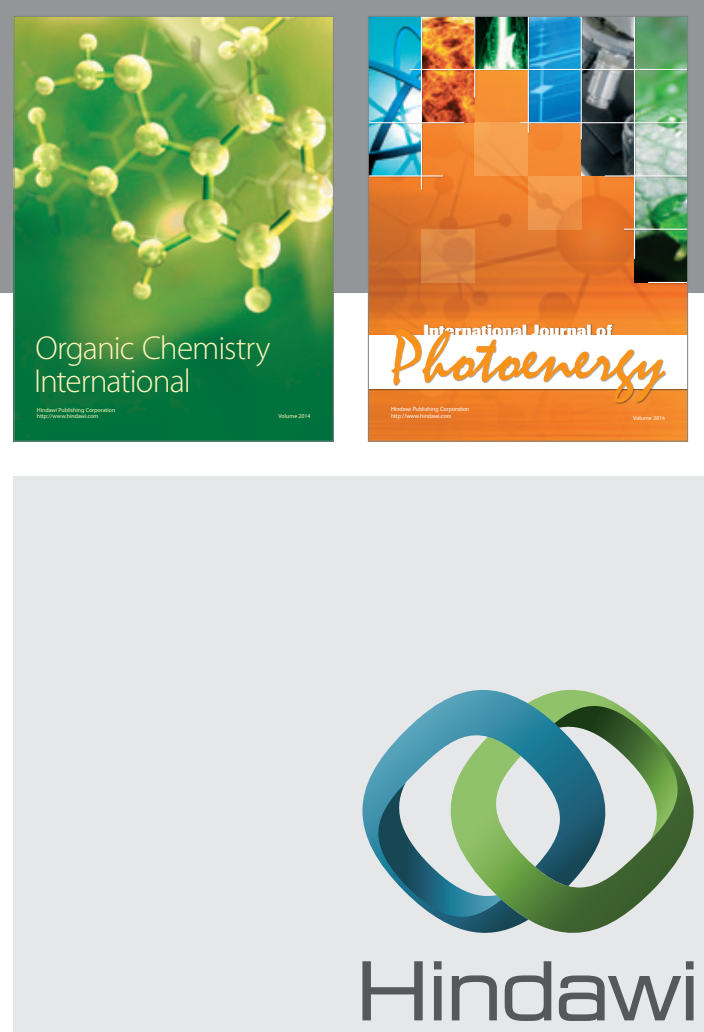

Submit your manuscripts at

http://www.hindawi.com

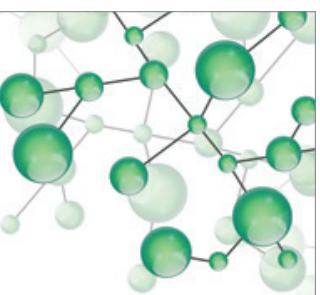

International Journal of

Inorganic Chemistry

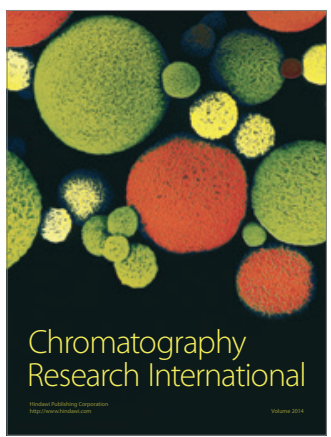

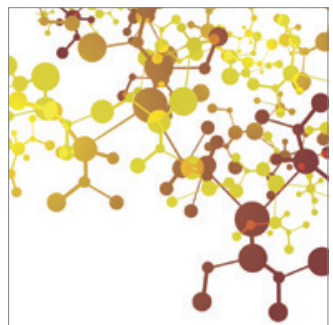

Applied Chemistry
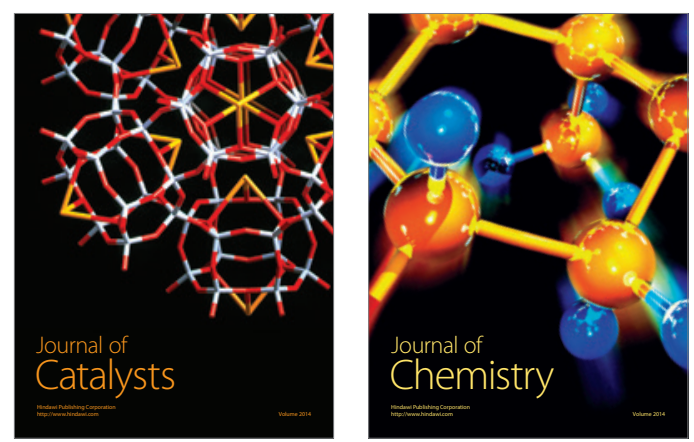
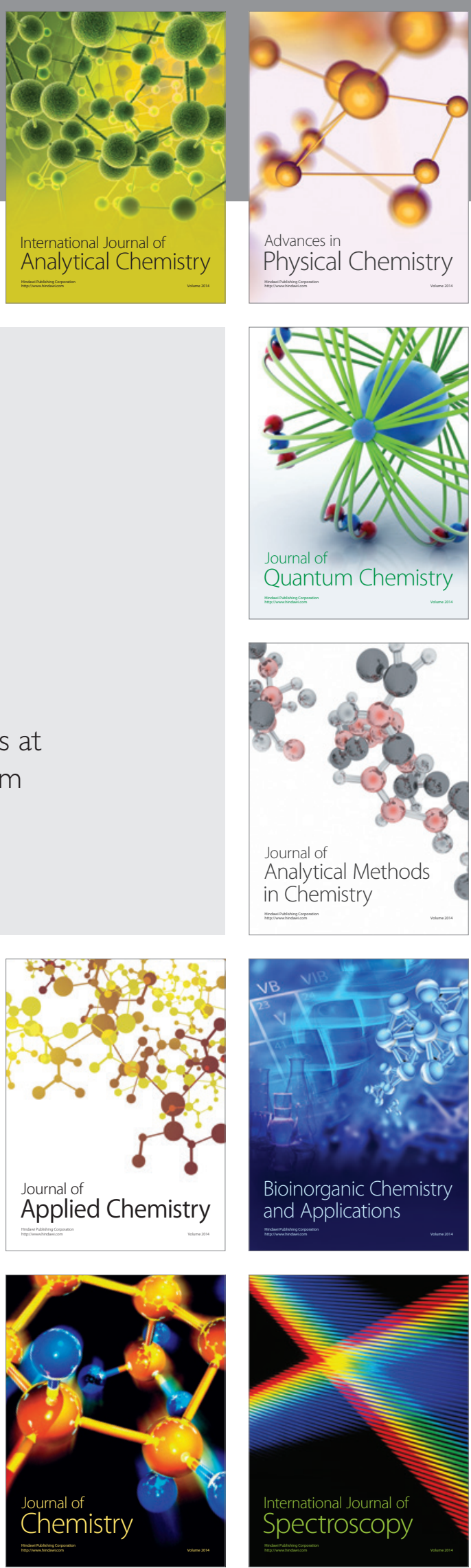